Supporting Information

\title{
Biomimetic Design of Peptide Neutralizer of Ebola Virus with Molecular Simulation
}

Qianqian Hou, Lin Zhang*

Department of Biochemical Engineering and Key Laboratory of Systems

Bioengineering of the Ministry of Education, School of Chemical Engineering and

Technology, Tianjin University, Tianjin 300350, China

Corresponding Author

*E-mail: linzhang@tju.edu.cn. Tel: +86 22 27403389. Fax: +86 2227403389. 
Table S1. EVINa of Each Peptide Candidate in the Library ${ }^{a}$

\begin{tabular}{|c|c|c|c|}
\hline Candidate & $E_{\mathrm{VINA}}(\mathrm{kcal} / \mathrm{mol})$ & Candidate & $E_{\mathrm{VINA}}(\mathrm{kcal} / \mathrm{mol})$ \\
\hline YFDWYGR & -7.6 & YFDWAIR & -6.5 \\
\hline YFDWGWR & -7.5 & YFDWDLR & -6.5 \\
\hline YFDWGPR & -7.4 & YFDWDVR & -6.5 \\
\hline YFDWPVR & -7.4 & YFDWDYR & -6.5 \\
\hline YFDWATR & -7.3 & YFDWEHR & -6.5 \\
\hline YFDWPFR & -7.3 & YFDWELR & -6.5 \\
\hline YFDWDFR & -7.2 & YFDWFER & -6.5 \\
\hline YFDWEPR & -7.2 & YFDWFNR & -6.5 \\
\hline YFDWGHR & -7.2 & YFDWGER & -6.5 \\
\hline YFDWHER & -7.2 & YFDWGLR & -6.5 \\
\hline YFDWHVR & -7.2 & YFDWGVR & -6.5 \\
\hline YFDWPPR & -7.2 & YFDWHGR & -6.5 \\
\hline YFDWTAR & -7.2 & YFDWHMR & -6.5 \\
\hline YFDWALR & -7.1 & YFDWITR & -6.5 \\
\hline YFDWAPR & -7.1 & YFDWKVR & -6.5 \\
\hline YFDWASR & -7.1 & YFDWLQR & -6.5 \\
\hline YFDWDAR & -7.1 & YFDWMFR & -6.5 \\
\hline YFDWDNR & -7.1 & YFDWNGR & -6.5 \\
\hline YFDWGYR & -7.1 & YFDWPMR & -6.5 \\
\hline YFDWIRR & -7.1 & YFDWQDR & -6.5 \\
\hline
\end{tabular}




\begin{tabular}{|c|c|c|c|}
\hline YFDWQER & -7.1 & YFDWQIR & -6.5 \\
\hline YFDWRGR & -7.1 & YFDWQMR & -6.5 \\
\hline YFDWVER & -7.1 & YFDWQVR & -6.5 \\
\hline YFDWYNR & -7.1 & YFDWQYR & -6.5 \\
\hline YFDWAAR & -7.0 & YFDWRER & -6.5 \\
\hline YFDWAYR & -7.0 & YFDWRIR & -6.5 \\
\hline YFDWDSR & -7.0 & YFDWRMR & -6.5 \\
\hline YFDWFIR & -7.0 & YFDWRSR & -6.5 \\
\hline YFDWGKR & -7.0 & YFDWRVR & -6.5 \\
\hline YFDWHQR & -7.0 & YFDWSHR & -6.5 \\
\hline YFDWIDR & -7.0 & YFDWSKR & -6.5 \\
\hline YFDWIHR & -7.0 & YFDWSWR & -6.5 \\
\hline YFDWKHR & -7.0 & YFDWTER & -6.5 \\
\hline YFDWMAR & -7.0 & YFDWYRR & -6.5 \\
\hline YFDWPER & -7.0 & YFDWYYR & -6.5 \\
\hline YFDWPGR & -7.0 & YFDWAWR & -6.6 \\
\hline YFDWPYR & -7.0 & YFDWDGR & -6.6 \\
\hline YFDWTDR & -7.0 & YFDWDHR & -6.6 \\
\hline YFDWTPR & -7.0 & YFDWDIR & -6.6 \\
\hline YFDWYAR & -7.0 & YFDWDPR & -6.6 \\
\hline YFDWYQR & -7.0 & YFDWEVR & -6.6 \\
\hline YFDWANR & -6.9 & YFDWFAR & -6.6 \\
\hline
\end{tabular}




\begin{tabular}{|c|c|c|c|}
\hline YFDWAQR & -6.9 & YFDWFPR & -6.6 \\
\hline YFDWAVR & -6.9 & YFDWFWR & -6.6 \\
\hline YFDWHPR & -6.9 & YFDWGQR & -6.6 \\
\hline YFDWLNR & -6.9 & YFDWHYR & -6.6 \\
\hline YFDWMPR & -6.9 & YFDWIAR & -6.6 \\
\hline YFDWNHR & -6.9 & YFDWIVR & -6.6 \\
\hline YFDWPAR & -6.9 & YFDWIWR & -6.6 \\
\hline YFDWPLR & -6.9 & YFDWKFR & -6.6 \\
\hline YFDWRYR & -6.9 & YFDWLAR & -6.6 \\
\hline YFDWSIR & -6.9 & YFDWLMR & -6.6 \\
\hline YFDWTTR & -6.9 & YFDWLVR & -6.6 \\
\hline YFDWVPR & -6.9 & YFDWLWR & -6.6 \\
\hline YFDWWKR & -6.9 & YFDWMIR & -6.6 \\
\hline YFDWDTR & -6.8 & YFDWPRR & -6.6 \\
\hline YFDWDWR & -6.8 & YFDWRAR & -6.6 \\
\hline YFDWFHR & -6.8 & YFDWRFR & -6.6 \\
\hline YFDWFLR & -6.8 & YFDWRNR & -6.6 \\
\hline YFDWGSR & -6.8 & YFDWRPR & -6.6 \\
\hline YFDWIIR & -6.8 & YFDWSSR & -6.6 \\
\hline YFDWMDR & -6.8 & YFDWSTR & -6.6 \\
\hline YFDWNAR & -6.8 & YFDWVHR & -6.6 \\
\hline YFDWNPR & -6.8 & YFDWVKR & -6.6 \\
\hline
\end{tabular}




\begin{tabular}{|c|c|c|c|}
\hline YFDWPDR & -6.8 & YFDWVNR & -6.6 \\
\hline YFDWPQR & -6.8 & YFDWVRR & -6.6 \\
\hline YFDWQLR & -6.8 & YFDWWFR & -6.6 \\
\hline YFDWRTR & -6.8 & YFDWWVR & -6.6 \\
\hline YFDWSGR & -6.8 & YFDWYDR & -6.6 \\
\hline YFDWSPR & -6.8 & YFDWYLR & -6.6 \\
\hline YFDWSVR & -6.8 & YFDWPNR & -6.7 \\
\hline YFDWTWR & -6.8 & YFDWPWR & -6.7 \\
\hline YFDWTYR & -6.8 & YFDWQNR & -6.7 \\
\hline YFDWWER & -6.8 & YFDWQQR & -6.7 \\
\hline YFDWAHR & -6.7 & YFDWSNR & -6.7 \\
\hline YFDWEFR & -6.7 & YFDWSQR & -6.7 \\
\hline YFDWEGR & -6.7 & YFDWVAR & -6.7 \\
\hline YFDWESR & -6.7 & YFDWVTR & -6.7 \\
\hline YFDWGDR & -6.7 & YFDWWPR & -6.7 \\
\hline YFDWGIR & -6.7 & YFDWWTR & -6.7 \\
\hline YFDWHFR & -6.7 & YFDWYKR & -6.7 \\
\hline YFDWHSR & -6.7 & YFDWYTR & -6.7 \\
\hline YFDWHTR & -6.7 & & \\
\hline YFDWIGR & -6.7 & & \\
\hline YFDWILR & -6.7 & & \\
\hline YFDWIPR & -6.7 & & \\
\hline
\end{tabular}




\begin{tabular}{ll}
\hline YFDWISR & -6.7 \\
YFDWKGR & -6.7 \\
YFDWLSR & -6.7 \\
YFDWNER & -6.7 \\
YFDWPHR & -6.7 \\
\hline
\end{tabular}

${ }^{a}$ only the candidates with $E_{\mathrm{VINA}} \leq-6.7 \mathrm{kcal} / \mathrm{mol}$ are shown herein. 
Table S2. RMSD of 173 Peptides Selected by EVINA

\begin{tabular}{|c|c|c|c|}
\hline Candidate & RMSD (nm) & Candidate & RMSD (nm) \\
\hline YFDWHMR & 0.19585 & YFDWPNR & 0.37879 \\
\hline YFDWTAR & 0.225 & YFDWPQR & 0.37927 \\
\hline YFDWDNR & 0.22812 & YFDWAWR & 0.37935 \\
\hline YFDWRYR & 0.24688 & YFDWWPR & 0.37964 \\
\hline YFDWMFR & 0.25302 & YFDWRFR & 0.37966 \\
\hline YFDWEGR & 0.25964 & YFDWGWR & 0.37977 \\
\hline YFDWRPR & 0.25998 & YFDWDPR & 0.38012 \\
\hline YFDWDLR & 0.26043 & YFDWSWR & 0.38095 \\
\hline YFDWAPR & 0.2609 & YFDWVRR & 0.38208 \\
\hline YFDWFWR & 0.26211 & YFDWPMR & 0.38251 \\
\hline YFDWAVR & 0.2634 & YFDWGHR & 0.3832 \\
\hline YFDWIIR & 0.26655 & YFDWHVR & 0.38365 \\
\hline YFDWRGR & 0.26936 & YFDWVPR & 0.3838 \\
\hline YFDWEVR & 0.26964 & YFDWGLR & 0.38385 \\
\hline YFDWTTR & 0.27242 & YFDWFER & 0.38515 \\
\hline YFDWRAR & 0.2728 & YFDWKVR & 0.3856 \\
\hline YFDWFAR & 0.2731 & YFDWGQR & 0.38628 \\
\hline YFDWPHR & 0.27444 & YFDWMDR & 0.38644 \\
\hline YFDWLNR & 0.27516 & YFDWIRR & 0.38689 \\
\hline YFDWDFR & 0.27616 & YFDWTWR & 0.38722 \\
\hline
\end{tabular}




\begin{tabular}{|c|c|c|c|}
\hline YFDWNPR & 0.27704 & YFDWALR & 0.38756 \\
\hline YFDWWKR & 0.27734 & YFDWYAR & 0.38843 \\
\hline YFDWMAR & 0.27747 & YFDWKGR & 0.38852 \\
\hline YFDWPGR & 0.27773 & YFDWSHR & 0.38861 \\
\hline YFDWEFR & 0.27831 & YFDWDTR & 0.38887 \\
\hline YFDWISR & 0.27998 & YFDWLVR & 0.38932 \\
\hline YFDWIPR & 0.28006 & YFDWTYR & 0.38986 \\
\hline YFDWNER & 0.28288 & YFDWESR & 0.38999 \\
\hline YFDWIVR & 0.28352 & YFDWPVR & 0.39113 \\
\hline YFDWFHR & 0.28387 & YFDWVHR & 0.39215 \\
\hline YFDWITR & 0.28599 & YFDWSGR & 0.39223 \\
\hline YFDWHQR & 0.28831 & YFDWDGR & 0.39231 \\
\hline YFDWASR & 0.29017 & YFDWGSR & 0.39261 \\
\hline YFDWNAR & 0.29177 & YFDWQVR & 0.39283 \\
\hline YFDWRIR & 0.29256 & YFDWEHR & 0.39297 \\
\hline YFDWDYR & 0.29281 & YFDWIHR & 0.39363 \\
\hline YFDWYQR & 0.29704 & YFDWLSR & 0.3946 \\
\hline YFDWRTR & 0.3004 & YFDWSTR & 0.39556 \\
\hline YFDWQYR & 0.30063 & YFDWIAR & 0.3966 \\
\hline YFDWPDR & 0.30209 & YFDWHYR & 0.39669 \\
\hline YFDWKFR & 0.3113 & YFDWTDR & 0.39804 \\
\hline YFDWRNR & 0.31335 & YFDWAHR & 0.39866 \\
\hline
\end{tabular}




\begin{tabular}{|c|c|c|c|}
\hline YFDWLAR & 0.31574 & YFDWMIR & 0.40062 \\
\hline YFDWAQR & 0.32246 & YFDWPLR & 0.40206 \\
\hline YFDWLMR & 0.33371 & YFDWQIR & 0.40378 \\
\hline YFDWFNR & 0.33444 & YFDWYTR & 0.40666 \\
\hline YFDWYRR & 0.33774 & YFDWANR & 0.40958 \\
\hline YFDWHPR & 0.3399 & YFDWNGR & 0.41033 \\
\hline YFDWWER & 0.34144 & YFDWDAR & 0.41113 \\
\hline YFDWVKR & 0.34331 & YFDWATR & 0.412 \\
\hline YFDWYYR & 0.34439 & YFDWGVR & 0.41307 \\
\hline YFDWYKR & 0.34664 & YFDWVTR & 0.41712 \\
\hline YFDWWVR & 0.35246 & YFDWQMR & 0.41716 \\
\hline YFDWSKR & 0.35299 & YFDWAIR & 0.4178 \\
\hline YFDWEPR & 0.35637 & YFDWGDR & 0.42271 \\
\hline YFDWDHR & 0.35671 & YFDWDWR & 0.42366 \\
\hline YFDWRMR & 0.35858 & YFDWPWR & 0.42479 \\
\hline YFDWELR & 0.35892 & YFDWVER & 0.42514 \\
\hline YFDWWFR & 0.3596 & YFDWGYR & 0.42527 \\
\hline YFDWGER & 0.35967 & YFDWVAR & 0.42623 \\
\hline YFDWPFR & 0.3597 & YFDWGPR & 0.42812 \\
\hline YFDWHER & 0.36 & YFDWRSR & 0.43199 \\
\hline YFDWAYR & 0.36034 & YFDWYGR & 0.4372 \\
\hline YFDWNHR & 0.36206 & YFDWSIR & 0.44017 \\
\hline
\end{tabular}




\begin{tabular}{|c|c|c|c|}
\hline YFDWYNR & 0.36338 & YFDWTPR & 0.44121 \\
\hline YFDWHSR & 0.36501 & YFDWPER & 0.44285 \\
\hline YFDWVNR & 0.36509 & YFDWPAR & 0.44455 \\
\hline YFDWSPR & 0.36567 & YFDWHFR & 0.44459 \\
\hline YFDWHTR & 0.36603 & YFDWPRR & 0.44543 \\
\hline YFDWQLR & 0.36688 & YFDWSVR & 0.45172 \\
\hline YFDWPYR & 0.36703 & YFDWGKR & 0.45326 \\
\hline YFDWDVR & 0.36769 & YFDWLWR & 0.45416 \\
\hline YFDWGIR & 0.36781 & YFDWFLR & 0.45493 \\
\hline YFDWRER & 0.36814 & YFDWDSR & 0.45504 \\
\hline YFDWIWR & 0.36843 & YFDWSNR & 0.45818 \\
\hline YFDWIDR & 0.37023 & YFDWRVR & 0.46 \\
\hline YFDWQER & 0.37035 & YFDWKHR & 0.46711 \\
\hline YFDWYLR & 0.37056 & YFDWFIR & 0.47711 \\
\hline YFDWILR & 0.37144 & YFDWWTR & 0.47769 \\
\hline YFDWQNR & 0.37291 & YFDWSSR & 0.49224 \\
\hline YFDWDIR & 0.37417 & YFDWYDR & 0.4971 \\
\hline YFDWPPR & 0.37458 & YFDWLQR & 0.49735 \\
\hline YFDWTER & 0.37469 & YFDWFPR & 0.49849 \\
\hline YFDWMPR & 0.37509 & YFDWIGR & 0.50614 \\
\hline YFDWQQR & 0.37528 & YFDWSQR & 0.50758 \\
\hline YFDWAAR & 0.37555 & YFDWHGR & 0.51528 \\
\hline
\end{tabular}


Table S3. Binding Free Energies (kcal/mol) of Four Neutralizers on GPcl

\begin{tabular}{cccccc}
\hline & $\Delta G_{\mathrm{vdw}}$ & $\Delta G_{\mathrm{SASA}}$ & $\Delta G_{\text {elec }}$ & $\Delta G_{\mathrm{PB}}$ & $\Delta G_{\text {bind }}$ \\
\hline YFDWHMR & $-18.9 \pm 10.8$ & $-3.5 \pm 1.2$ & $-141.9 \pm 34.1$ & $132.7 \pm 30.5$ & $-31.5 \pm 15.5$ \\
YFDWRYR & $-31.6 \pm 10.3$ & $-5.2 \pm 1.0$ & $-284.3 \pm 34.2$ & $264.0 \pm 21.3$ & $-57.1 \pm 19.6$ \\
YFDWDNR & $-40.7 \pm 6.3$ & $-5.2 \pm 0.7$ & $-52.9 \pm 20.9$ & $90.4 \pm 13.4$ & $-8.4 \pm 2.9$ \\
YFDWTAR & $-29.0 \pm 7.5$ & $-4.6 \pm 0.8$ & $-140.1 \pm 31.5$ & $146.0 \pm 33.9$ & $-27.7 \pm 2.5$ \\
\hline
\end{tabular}



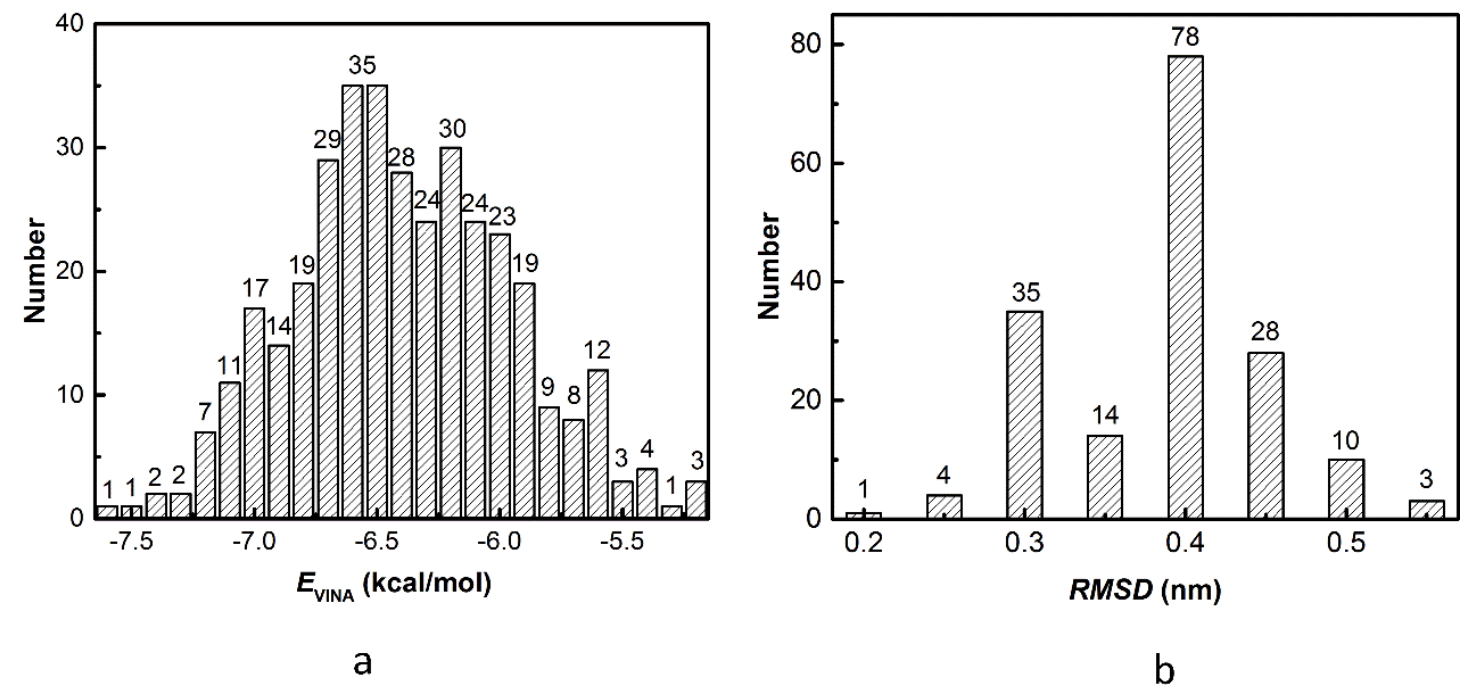

Figure S1. Distribution of docking scores by VINA (a) and RMSD (b). 

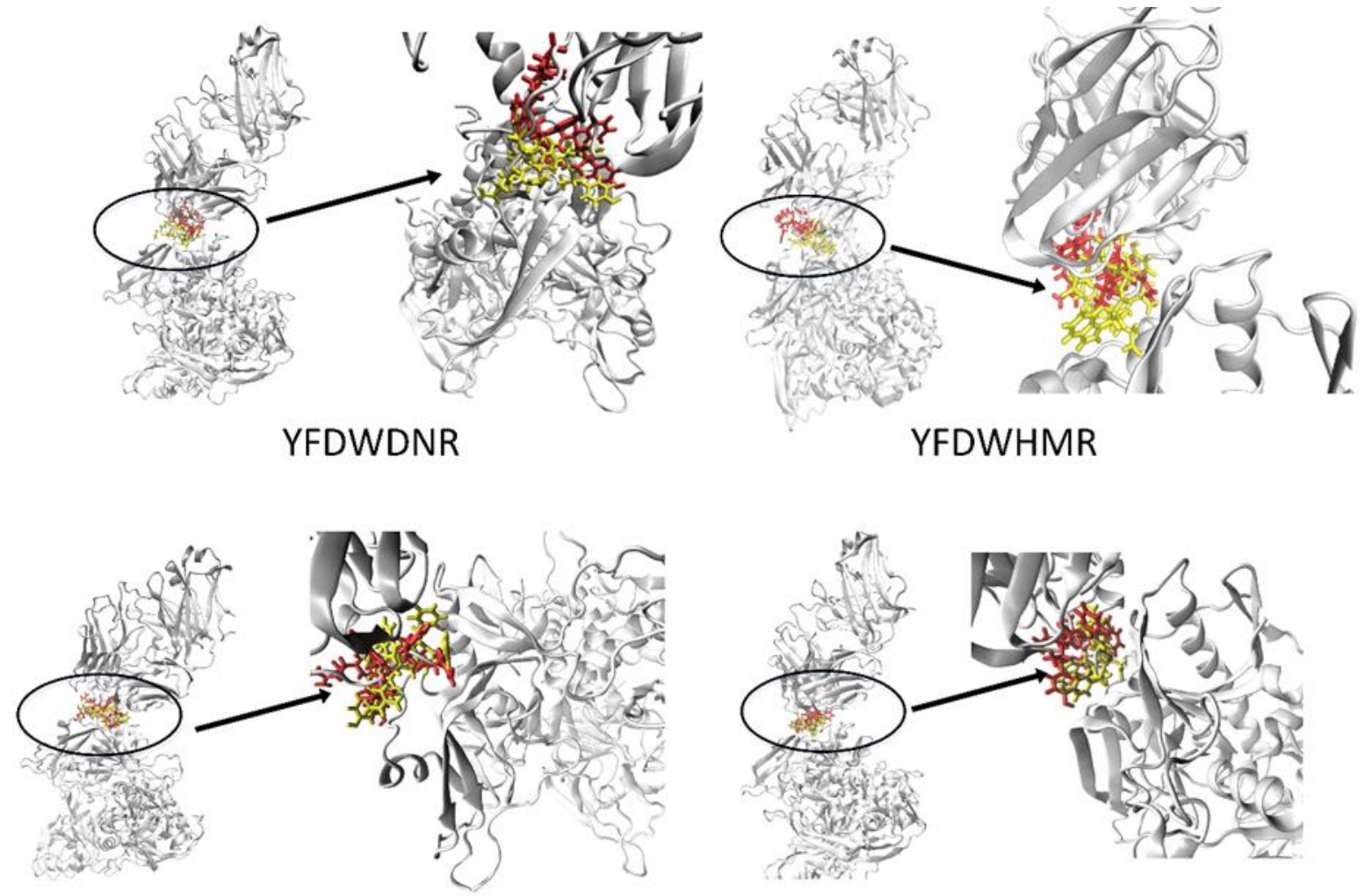

YFDWRYR

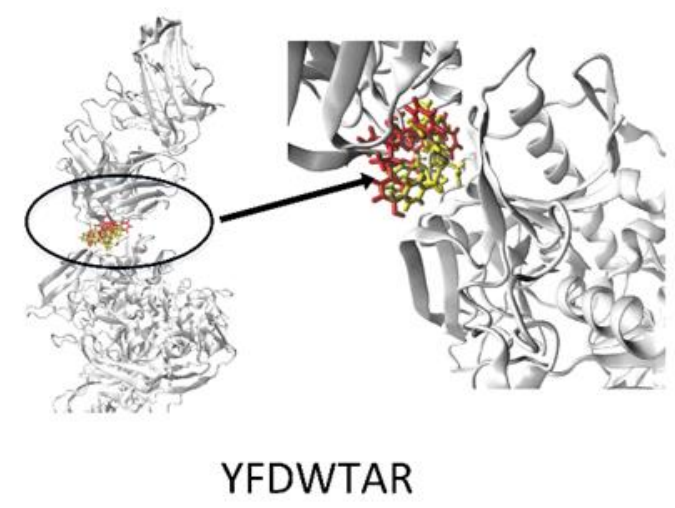

Figure S2. The superposition of the docked conformation (red) of selected candidate to the original structure (yellow) in the affinity binding model. 


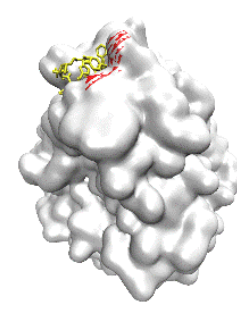

YFDWHMR

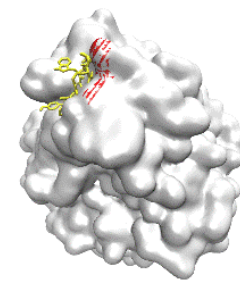

YFDWTAR

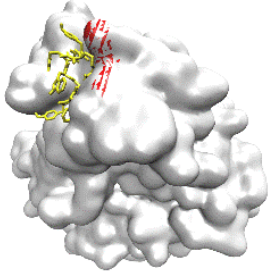

YFDWRYR

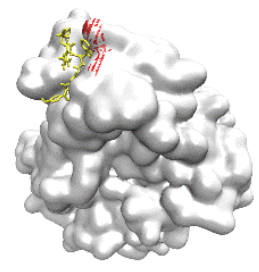

YFDWDNR

Figure S3. Conformation of four candidates binding on GPcl as compared with the predicted binding sites. 

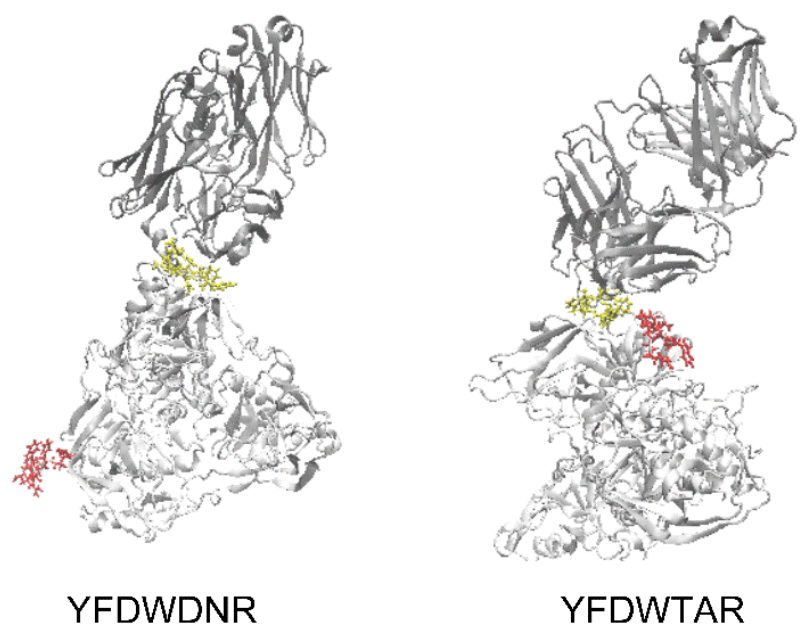

Figure S4. Comparison of the final binding conformation of YFDWDNR and YFDWTAR in MD simulations (red) to the original structure in ABM (yellow). 


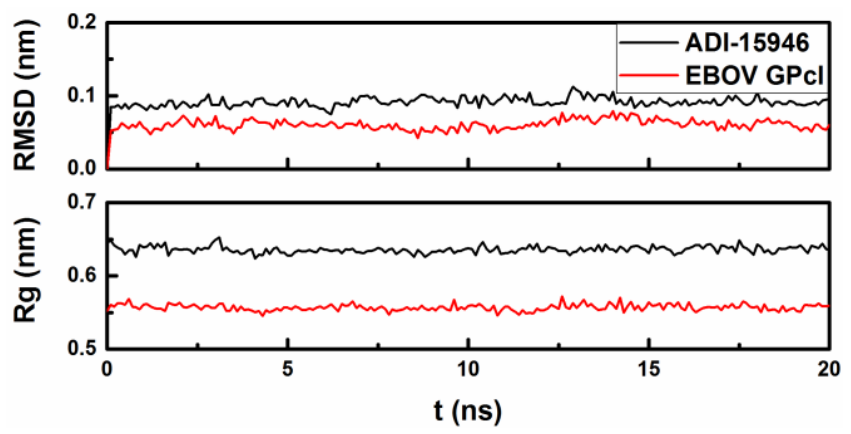

Figure S5. Time courses of RMSD and Rg of the key amino acids of the binding site in GPcl and ADI-15946. 